Compte-rendu de Richard Heffernan, Colin Hay, Meg Russell, Philip Cowley (dirs), Developments in British Politics 10, Londres, Palgrave, $10^{\mathrm{e}}$ édition, 2016

\title{
Thibaud Harrois
}

\author{
CpenEdition \\ Journals \\ Édition électronique \\ URL : http://journals.openedition.org/rfcb/1245 \\ DOI : $10.4000 /$ rfcb. 1245 \\ ISSN : 2429-4373 \\ Éditeur \\ CRECIB - Centre de recherche et d'études en civilisation britannique \\ Référence électronique \\ Thibaud Harrois, « Compte-rendu de Richard Heffernan, Colin Hay, Meg Russell, Philip Cowley (dirs), \\ Developments in British Politics 10, Londres, Palgrave, 10 édition, $2016 »$, Revue Française de Civilisation \\ Britannique [En ligne], XXII-2 | 2017, mis en ligne le 30 mai 2016, consulté le 24 avril 2019. URL : http:// \\ journals.openedition.org/rfcb/1245; DOI : 10.4000/rfcb.1245
}

Ce document a été généré automatiquement le 24 avril 2019

\section{(i) 89

Revue française de civilisation britannique est mis à disposition selon les termes de la licence Creative Commons Attribution - Pas d'Utilisation Commerciale - Pas de Modification 4.0 International. 


\title{
Compte-rendu de Richard Heffernan, Colin Hay, Meg Russell, Philip Cowley (dirs), Developments in British Politics 10, Londres, Palgrave, $10^{\mathrm{e}}$ édition, 2016
}

\author{
Thibaud Harrois
}

\section{RÉFÉRENCE}

Richard Heffernan, Colin Hay, Meg Russell, Philip Cowley (dirs), Developments in British Politics 10, Londres, Palgrave, $10^{e}$ édition, 2016, ISBN : 9781137494733, 376p.

1 Cette édition de Developments in British Politics, dirigée par Richard Heffernan, Colin Hay, Meg Russell et Philip Cowley, rassemble des contributions rédigées après les élections britanniques de 2015 et achevées aux lendemains du référendum du 23 juin 2016 sur l'appartenance du Royaume-Uni à l'Union européenne (UE). L'objectif des dix-sept auteurs ayant participé à la rédaction des quinze chapitres que comporte l'ouvrage est premièrement d'analyser les évolutions de la vie institutionnelle et politique britannique entre l'arrivée au pouvoir de David Cameron en 2010 et sa démission après qu'il a échoué à convaincre une majorité d'électeurs britanniques de voter en faveur du maintien dans l'UE. Mais la dixième édition des Developments in British Politics est également l'occasion de porter les regards plus loin dans le passé en s'interrogeant sur ce qui fait la spécificité de la politique britannique en 2016, en comparaison notamment avec le tableau qui en était dressé dans la première édition de la série, publiée en 1983 (à ce propos, voir le chapitre 15, rédigé par Colin Hay).

2 Dans le chapitre liminaire, Andrew Gamble présente les caractéristiques du système politique britannique et décrit la crise que traverse le pays, thème qui traverse l'ensemble 
de l'ouvrage. En effet, l'unité territoriale du Royaume-Uni, la légitimité de ses institutions politiques nationales ou bien la place qu'il occupe sur la scène internationale sont profondément remises en question. Certes, aucun de ces phénomènes ne constitue une question véritablement nouvelle. Mais la montée des nationalismes, la fin de la prééminence des partis conservateur et travailliste sur la vie politique et les incertitudes sur la relation qu'entretiendra à l'avenir la Grande-Bretagne avec ses partenaires européens ont été considérablement renforcées au cours des cinq dernières années. Derrière l'apparente stabilité $d u$ système politique du Royaume-Uni, l'identité britannique et toute l'organisation de la vie politique du pays apparaissent troublées.

3 La stabilité des institutions politiques britanniques a été démontrée par leur capacité à s'adapter à l'arrivée au pouvoir d'une coalition formée par les conservateurs et les libéraux-démocrates en mai 2010. Comme le soulignent Ben Yong et Tim Bale (chapitre 2), alors que Whitehall est peu habitué à la formation de coalitions, les ministères ont su s'adapter au fonctionnement imposé par ce type de gouvernement. Mais ces mêmes ministères, lieux de décision clefs dans la conduite de la politique nationale, ont également dû se plier à de nouvelles contraintes dans un contexte de réforme de la fonction publique. En effet, Catherine Haddon (chapitre 9) montre comment depuis 2010 le gouvernement a œuvré en vue de la modernisation et de la rationalisation de l'administration, en particulier au sein de Whitehall, afin de répondre à l'exigence d'une importante réduction des dépenses publiques. Même si des inquiétudes ont pu être exprimées (en particulier concernant la manière de maintenir l'indépendance des hautsfonctionnaires), il semblerait que ce soit la continuité et la stabilité qui aient prévalu lors de la mise en œuvre de ces réformes.

Le Parlement a également été caractérisé par une remarquable stabilité. L'adoption de la loi de 2011 fixant la durée d'une mandature à cinq ans y a certainement contribué, puisque le Premier ministre n'a désormais plus la possibilité de dissoudre la Chambre des Communes au moment qui lui semble le plus opportun pour permettre à son parti de remporter une majorité des sièges. Autre élément de stabilité, Meg Russell (chapitre 6) rappelle qu'entre 2010 et 2015, toutes les tentatives de réformer le mode de scrutin ou le fonctionnement du Parlement ont échoué. Dans l'enceinte de celui-ci, il y eut peu d'affrontements entre partenaires de coalition. Cela ne signifie cependant pas pour autant que le Parlement se soit contenté de suivre la politique définie par le gouvernement. $\mathrm{Au}$ contraire, Westminster a pesé sur le contenu des débats et la formulation des politiques, allant jusqu'à forcer le gouvernement à revoir ses décisions, principalement sous l'influence de "backbenchers» conservateurs, dont le poids a été renforcé depuis l'élection de 2015 en l'absence d'une opposition unie autour de son chef de file.

5 Toutefois, malgré cette apparente stabilité des institutions politiques britanniques, cellesci traversent une crise de légitimité due à leur manque de représentativité. C'est particulièrement le cas du Parlement où, comme l'explique Alan Renwick (chapitre 3), la prééminence des partis conservateur et travailliste, due au mode de scrutin uninominal majoritaire à un tour qui leur donne un avantage en nombre de sièges à la Chambre des Communes, masque en réalité une dispersion des voix des électeurs beaucoup plus grande aujourd'hui qu'elle ne l'était jusque dans les années 1970. De plus, Philip Cowley souligne que, malgré les progrès obtenus en vue d'une meilleure représentation des femmes ainsi que des minorités (ethniques ou sexuelles par exemple), ceux-ci sont encore insuffisants pour diversifier une classe politique encore largement homogène et perçue comme 
détachée de la réalité de la population britannique (chapitre 7). Symptôme de la rupture entre le peuple et ceux qui se proposent de le représenter, le taux de participation aux élections n'a cessé de diminuer depuis le dernier quart $\mathrm{du} \mathrm{xx}^{\mathrm{e}}$ siècle. Même la multiplication de formes "non-conventionnelles " d'engagement politique, présentées par Maria Grasso (chapitre 8), n’a pas enrayé la désaffection, voire le désintérêt, de la population, y compris des jeunes, pour la chose publique.

6 Ce phénomène se remarque également dans l'évolution des médias. L'apparition et la multiplication de nouveaux moyens d'information (principalement en ligne) ont altéré la manière dont ces informations sont produites et consommées. Toutefois, Richard Heffernan (chapitre 10) remarque que les médias ont une capacité limitée à modifier l'opinion que les électeurs se font des personnalités politiques de premier plan. De plus, il n'existe pas aujourd'hui un auditoire beaucoup plus large que celui qu'atteignaient déjà les médias traditionnels en recul (journaux imprimés et télévision). Ainsi l'utilisation de nouveaux moyens de communication pour s'adresser aux électeurs ne permet pas véritablement de freiner l'indifférence d'un grand nombre d'entre eux pour un débat public vu comme largement dépolitisé.

7 A ce titre, le chapitre consacré par Deborah Mabbett à la mise en œuvre de la politique d'austérité est particulièrement parlant (chapitre 11). Malgré les résultats mitigés des mesures prises par le gouvernement de coalition pour réduire les déficits publics, l'austérité n'a pas été fondamentalement remise en question par l'opposition travailliste. Les électeurs se trouvèrent ainsi davantage appelés à choisir le parti auquel ils accorderaient leur confiance pour réduire les déficits qu'à se prononcer devant une véritable alternative en termes de politique économique (seule l'élection de Jeremy Corbyn a fait ouvertement entrer le parti travailliste dans l'opposition à la politique d'austérité).

Contrairement aux deux grands partis, les plus petites formations connaissent une hausse des adhésions, même si l'augmentation du nombre de voix qu'elles récoltent ne se traduit pas toujours par un plus grand nombre d'élus, en fonction du mode de scrutin en vigueur aux différentes élections (chapitres 3, 4 et 5). Parmi ces partis, le Scottish National Party a vu sa popularité croître depuis la mise en œuvre de la "devolution » jusqu'à obtenir une majorité des sièges au Parlement écossais, ce qui lui a permis d'obtenir l'organisation d'un référendum sur l'indépendance de l'Ecosse, étudié en détails par Charlie Jeffery (chapitre 13). Malgré l'échec du camp indépendantiste, le SNP est parvenu à s'imposer comme la première force politique écossaise, y compris au sein du Parlement britannique aux dépens du parti travailliste. Cette montée du nationalisme n'est pas limitée à l'Ecosse. $\mathrm{Au}$ Pays de Galles, en Irlande du Nord, mais aussi en Angleterre, le référendum de septembre 2014 a conduit à une révision constitutionnelle accordant davantage de pouvoirs à chacune des nations. Cette fragmentation du territoire britannique a été encore renforcée par le constat de profondes divergences sur la question européenne lors du référendum du 23 juin 2016.

9 L'analyse du débat sur l'appartenance du Royaume-Uni à l'UE proposée par Andrew Geddes (chapitre 14) retrace l'évolution de l'euroscepticisme. D'abord expression du refus d'être entraîné dans un projet politique supranational qui dépasse le cadre du seul marché commun, l'opposition à l'UE a rencontré un succès populaire croissant que les crises économiques et migratoires de ces dernières années ont renforcé. Le succès des partisans de la sortie de l'UE s'explique ainsi notamment par la manière dont ils ont su utiliser la méfiance d'une partie grandissante de la population vis-à-vis de la politique 
d'immigration (dont les enjeux sont présentés par Maria Sobolewska et Robert Ford dans le chapitre 12), auquel s'est ajouté un rejet des élites qui s'est exprimé principalement parmi les électeurs les plus âgés, les moins éduqués et les plus pauvres. L'ouvrage, publié quelques semaines après le référendum, ne peut proposer une analyse complète des conséquences de ce vote. Toutefois, il apparaît déjà clairement que les doutes qui y sont exprimés sur l'avenir de l'identité, du territoire et des institutions britanniques n'en seront que plus profonds dans les prochaines années.

Extrêmement utile du fait de la qualité des chapitres et de la diversité des sujets abordés, cet ouvrage ne constitue cependant qu'un panorama général, et incomplet, des évolutions de la politique britannique depuis 2010. On regrette quelques redondances (l'analyse du succès croissant du UKIP ou du SNP entre autres) alors que d'autres aspects, comme les évolutions des politiques publiques, auraient pu être davantage détaillés (à titre d'exemple, les débats sur les politiques de logement ou d'éducation ne sont presque pas abordés) et que la réflexion sur la politique étrangère est cantonnée presque exclusivement à la question de la relation avec l'UE. Les références bibliographiques proposées permettront néanmoins au lecteur d'approfondir les réflexions entamées par ce qui constitue, comme les éditions précédentes, un manuel de référence pour tous ceux qui s'intéressent à la politique du Royaume-Uni.

\section{AUTEURS}

\section{THIBAUD HARROIS}

Université Paris 1 Panthéon-Sorbonne 